\title{
Padronização de parâmetros ecocardiográficos de cães da raça Golden Retriever clinicamente sadios
}

\author{
Standardization of echocardiographic parameters of healthy Golden Retriever dogs \\ Arine Pellegrino $^{\mathrm{I}}$ Lilian Caram Petrus ${ }^{\mathrm{II}}$ Guilherme Gonçalves Pereira ${ }^{\mathrm{II}}$ \\ Elaine Cristina Soares ${ }^{\mathrm{II}}$ Ronaldo Jun Yamato ${ }^{\mathrm{II}}$ Eduardo Lipparelli Fernandez ${ }^{\mathrm{I}}$ \\ Maria Helena Matiko Akao Larsson ${ }^{\text {IV }}$
}

RESUMO

A distrofia muscular de Duchenne (DMD) em humanos é uma alteração neuromuscular hereditária, de caráter recessivo, ligada ao cromossomo X e causada pela ausência ou disfunção da distrofina. Clinicamente, caracterizase por severa alteração na musculatura esquelética, resultando em morte precoce do indivíduo acometido. Em cães da raça Golden Retriever, a mutação que leva à distrofia muscular ocorre espontaneamente e a extensa homologia entre a patogênese da DMD e da distrofia muscular do Golden Retriever permite qualificar o cão como principal substituto de humanos nos testes clínicos de novas terapias. O miocárdio deficiente em distrofina é mais vulnerável à sobrecarga de pressão e os pacientes com DMD podem desenvolver cardiomiopatia dilatada e hipertensão arterial; à ecocardiografia, verificamse anormalidades na função diastólica, além de insuficiência sistólica em alguns pacientes mais velhos. No presente estudo, 41 cães da raça Golden Retriever, clinicamente sadios, foram submetidos ao exame ecocardiográfico com a finalidade de se obterem os citados parâmetros na referida raça, o que futuramente poderá servir de referência na identificação de cães portadores ou afetados pela distrofia muscular.

Palavras-chave: ecocardiografia, distrofia muscular do Golden Retriever (GRMD), distrofia muscular de Duchenne (DMD), cardiomiopatia de Duchenne, cardiomiopatia.

\section{ABSTRACT}

The Duchenne's muscular dystrophy (DMD) in humans is a recessive $X$-linked neuromuscular disease, caused either by the absence or dysfunction of the dystrophin. Clinically it is characterized by severe alteration in the skeletal musculature, resulting in precocious death of the affected patient. In Golden Retriever dogs, the mutation that determines the muscular dystrophy occurs spontaneously and the extensive homology among the pathogenesis of DMD and of Golden Retriever muscular dystrophy allows to qualify the dog as the main substitute of humans in the clinical tests of new therapies. The deficient myocardium in dystrophin is more vulnerable to the pressure overload, and the patients with DMD can develop dilated cardiomyopathy as well as arterial hypertension; in echocardiography, diastolic function abnormalities are verified and systolic inadequacy can be observed in some old patients. In the present study, 41 healthy Golden Retriever dogs were evaluated by echocardiographic exam with the purpose to obtain parameters for the standardization of these cardiovascular characteristics in the refered breed, what hereafter can be used as reference in the identification of bearer or affected dogs by muscular dystrophy.

Key words: echocardiography, Golden Retriever muscular dystrophy (GRMD), Duchenne's muscular dystrophy (DMD), Duchenne's cardiomyopathy, cardiomyopathy.

\section{INTRODUÇÃO}

A distrofia muscular de Duchenne (DMD) é uma alteração neuromuscular hereditária comum em humanos; afeta, aproximadamente, um em cada 3.500 indivíduos do sexo masculino com herança ligada ao cromossomo $\mathrm{X}$ e é caracterizada por contínua necrose muscular e degeneração, com eventual fibrose e

\footnotetext{
IHospital Veterinário (HOVET), Faculdade de Medicina Veterinária e Zootecnia, Universidade de São Paulo (FMVZ-USP), São Paulo, SP, Brasil.

IIAutônomo, São Paulo, SP, Brasil.

"IIIPrograma de Pós-graduação do Departamento de Clínica Médica, FMVZ-USP, São Paulo, SP, Brasil

IV Departamento de Clínica Médica, FMVZ-USP. Av. Orlando Marques Paiva, 87, 05508-900, São Paulo, SP, Brasil. E-mail: akaolar@usp.br. Autor para correspondência.
} 
infiltração por tecido adiposo (MOISE et al., 1991; HOWELL et al., 1997). É de caráter recessivo e causada pela ausência ou disfunção da distrofina, proteína encontrada numa variedade de tecidos, principalmente nas musculaturas esquelética e cardíaca, nervos e regiões específicas do sistema nervoso central. Clinicamente, caracteriza-se por severa alteração na musculatura esquelética, resultando em morte precoce do indivíduo acometido (ANDERSON et al., 2002; CHILDERS et al., 2002; HAINSEY et al., 2003; PACIORETTY et al., 1994).

Camundongos e cães com distrofia muscular, ambos com defeito na distrofina dos tecidos musculares, são modelos para a DMD em humanos (COZZI et al., 2001; NGUYEN et al., 2002). Porém, os cães constituem-se nas melhores fenocópias da doença em humanos, quando comparados com outros modelos animais (BOGDANOVICH et al., 2003; COLLINS \& MORGAN, 2003). A distrofia muscular canina ligada ao cromossomo $\mathrm{X}$ é reconhecida e mais bem caracterizada em cães da raça Golden Retriever, nos quais ocorre de forma espontânea (DANI et al., 2000; SHIMATSO et al., 2003). Colônias de cães com distrofia muscular foram estabelecidas e estudos sobre genética, clínica, patogenia, biologia molecular e imunocitoquímica realizados no modelo canino são úteis para avaliar a distrofia muscular de Duchenne em humanos (VALENTINE et al., 1992; DANI et al., 2000).

No homem, alterações cardíacas são complicações reconhecidas em portadores adultos de distrofinopatias ligadas ao cromossomo $\mathrm{X}$ (HOOGERWAARD et al., 1999; NOLAN et al., 2003). O miocárdio deficiente em distrofina é mais vulnerável à sobrecarga de pressão e os pacientes com DMD podem desenvolver cardiomiopatia dilatada e hipertensão arterial (HUNSAKER et al., 1982; KAMOGAWA et al., 2001).

Em cães, a cardiomiopatia associada à distrofia muscular de Duchenne é caracterizada por fibrose progressiva no epicárdio e miocárdio, primeiramente na parede livre posterobasal do ventrículo esquerdo (CZINER \& LEVIN, 1993). Sinais clínicos de insuficiência cardíaca congestiva só são verificados em período próximo à morte (MOISE et al., 1991). À ecocardiografia, verificam-se anormalidades na função diastólica e pode-se observar insuficiência sistólica em alguns pacientes mais idosos (HUNSAKER et al., 1982; MOISE et al., 1991). À ecocardiografia bidimensional, verificam-se lesões hiperecóicas que correspondem à calcificação do miocárdio e à presença de tecido conjuntivo denso ao redor.
Segundo CHETBOUL et al. (2004), o diagnóstico precoce da cardiomiopatia dilatada de Duchenne pode ser realizado por meio do "Tissue Doppler Imaging” (TDI), ecocardiografia capaz de detectar alterações precoces na função do miocárdio em cães da raça Golden Retriever. Cães com distrofia muscular apresentam, no TDI, diminuição no gradiente de velocidade miocárdica sistólica e alteração no gradiente de velocidade miocárdica diastólica. O TDI é mais sensível que a ecocardiografia convencional, sendo capaz de detectar anormalidades miocárdicas pré-clínicas, antes da ocorrência da dilatação do ventrículo esquerdo e do estabelecimento da disfunção cardíaca.

Para que se possam avaliar as alterações cardíacas presentes em cães da raça Golden Retriever afetados ou portadores do gene da distrofia muscular, há necessidade do conhecimento dos parâmetros ecocardiográficos considerados normais para esta raça. O Golden Retriever tem-se tornado bastante comum no Brasil nos últimos anos, apresentando-se como uma raça em ascensão. Devido ao aumento de cruzamentos entre animais de linhagens diversas e sabendo-se da possibilidade de ocorrência de mutações espontâneas que podem levar à distrofia muscular, é de fundamental importância o conhecimento de parâmetros cardiovasculares normais para a referida raça. Portanto, a finalidade do presente estudo foi a obtenção de parâmetros para contribuir com a padronização do ecocardiograma de cães sadios da raça Golden Retriever, para que possam servir de referência.

\section{MATERIAL E MÉTODOS}

Animais e grupos experimentais

Cães sadios da raça Golden Retriever, provenientes do canil GRMD da Faculdade de Medicina Veterinária e Zootecnia da Universidade de São Paulo (FMVZ-USP), de canis particulares de São Roque, Itu, Carapicuíba e Granja Viana e de proprietários particulares foram utilizados no presente trabalho. Todos os animais selecionados foram submetidos a exames físico, hematológico e bioquímico, previamente à inclusão no projeto.

Os animais do estudo em questão foram subdivididos em dois grupos: grupo I: animais de até 12 meses de idade; grupo II: animais com idades entre 12 e 36 meses.

Devido à grande variação de peso e porte entre os animais do grupo I, composto por filhotes em fase de desenvolvimento, fez-se necessária a subdivisão desta categoria em: grupo I-1: animais de até três meses de idade - $0 \vdash 3$; grupo I-2: animais entre três e seis 
meses de idade - 3 I-6; grupo I-3: animais entre seis e nove meses de idade - 6 I-9; grupo I-4: animais entre nove e 12 meses de idade - 9।-12.

\section{Genotipagem}

Apenas os cães procedentes do canil GRMD da FMVZ-USP, logo após o nascimento, foram identificados como "sadios" quanto à distrofia muscular por meio do "kit” GFX Genomic Blood DNA Purification, em exame realizado no Centro de Estudos do Genoma Humano do Instituto de Biociências da Universidade de São Paulo.

\section{Exame ecocardiográfico}

Para a realização do exame ecocardiográfico, os animais foram posicionados em decúbito lateral esquerdo com o transdutor sobre o tórax, para a obtenção das imagens através da janela paraesternal direita e paraesternal esquerda cranial e caudal. Uma grossa camada de gel era aplicada entre a pele do animal e o transdutor, para diminuir a interferência que o ar exerce no exame ultra-sonográfico.

O exame ecocardiográfico foi realizado conforme recomendações da Echocardiography Committee of the Specialty of Cardiology-American College of Veterinary Internal Medicine (THOMAS et al., 1993) e American Society of Echocardiography (ASE) (BOON, 1998; YAMATO, 2006). Os parâmetros em modo $\mathrm{M}$ avaliados e calculados, de acordo com BOON (1998) e YAMATO (2006), foram: diâmetro interno da cavidade do ventrículo esquerdo na diástole (DVEd); diâmetro interno da cavidade do ventrículo esquerdo na sístole (DVEs); espessura do septo interventricular na diástole (SIVd); espessura do septo interventricular na sístole (SIVs); espessura da parede do ventrículo esquerdo na diástole (PVEd); espessura da parede do ventrículo esquerdo na sístole (PVEs); raiz da artéria aorta (Ao); átrio esquerdo (AE); tempo de ejeção do ventrículo esquerdo (TEVE); fração de ejeção do ventrículo esquerdo (FE); freqüência cardíaca (FC); relação entre a medida do átrio esquerdo e a raiz da aorta (AE/Ao); relação entre a medida da espessura do septo interventricular e a espessura da parede do ventrículo esquerdo na diástole (SIVd/ PVEd); relação entre a medida da espessura do septo interventricular e a espessura da parede do ventrículo esquerdo na sístole (SIVs/PVEs); distância entre o ponto E da valva mitral e o septo interventricular (dES); velocidade de encurtamento circunferencial da fibra miocárdica (Vecf); engrossamento sistólico da parede livre do ventrículo esquerdo (PPVE); engrossamento sistólico do septo interventricular (PSIV); fração de encurtamento (FS). Também foram avaliados débito cardíaco (DC), volume na sístole (VOLs), volume na diástole (VOLd) e volume de ejeção (Vej); e parâmetros de ecocardiografia Doppler como fluxo mitral-onda E (mitral E), fluxo mitral - onda A (mitral A), aceleração mitral (aceleração), velocidade máxima do fluxo aórtico (Ao-Vmáx), aceleração aórtica (Ao-TA), gradiente de pressão aórtico (Ao-Grad), velocidade máxima do fluxo pulmonar (Ap-Vmáx), gradiente de pressão pulmonar (Ap-Grad), e tempo de relaxamento isovolumétrico (TRIV).

Realizou-se exame ecocardiográfico em 21 animais do grupo I (cinco cães em cada subgrupo I-1, I-2 e I-3; e seis no subgrupo I-4); e em 20 animais do grupo II.

Análise dos Resultados

Os valores dos parâmetros ecocardiográficos obtidos e apresentados sob a forma de média e dois desvios-padrão foram analisados, posteriormente, por meio do programa “GraphPad InStat"- teste de Bonferroni.

\section{RESULTADOS E DISCUSSÃO}

\section{Exame Ecocardiográfico}

Todos os animais do grupo I apresentaram valores normais na ecocardiografia modo $\mathrm{M}$, segundo WENDY (2003) (Tabelas 1 e 2). Inicialmente, pôde-se verificar que a freqüência cardíaca é mais alta nos animais mais jovens e diminui com a idade. Os parâmetros relacionados aos diâmetros e espessuras de cavidades e estruturas (DVEd, DVEs, SIVd, SIVs, PVEd, PVEs, Ao, AE) (Tabela 1) e os volumes sangüíneos (VOLd, VOLs e Vej) (Tabela 2) apresentaram aumento progressivo conforme a idade e o porte dos cães. Estatisticamente, verificaram-se diferenças significativas quanto aos parâmetros de ecocardiografia modo M: DVEd, DVEs, SIVd, SIVs, PVEd, PVEs, Ao, AE, dES $(\mathrm{P}<0,001)$; quanto aos volumes sangüíneos: VOLd, VOLs e Vej $(\mathrm{P}=<0,001)$; e quanto ao valor da aceleração do fluxo aórtico $(\mathrm{P}<0,05)$, nos diferentes subgrupos e grupos. Porém, apesar da variação etária e de peso entre os indivíduos do grupo I, as relações (SIVd/PVEd, SIVs/PVEs) e FE, Vecf, PPVE, PSIV, FS e DC mantiveram-se praticamente constantes nos animais dos diferentes subgrupos (Tabelas 1 e 2).

Não foram observadas diferenças estatísticas entre os subgrupos e grupos quanto aos parâmetros de ecocardiografia Doppler e quanto aos parâmetros SIVd/PVEd, SIVs/PVEs, FE, Vecf, PPVE, PSIV, FS e DC (P>0,05) (Tabelas 1 e 2).

Segundo FOX et al. (1999), um cão da raça Golden Retriever adulto, de peso médio de 32 (23-41)kg, 
Tabela 1 - Média e desvio padrão de parâmetros ecocardiográficos de cães da raça Golden Retriever, clinicamente normais - São Paulo 2005.

\begin{tabular}{|c|c|c|c|c|c|c|c|c|c|c|}
\hline Grupos & Peso (kg) & $\begin{array}{c}\text { Idade } \\
\text { (meses) }\end{array}$ & FC & DVEd & DVEs & SIVd & SIVs & PVEd & PVEs & Ao \\
\hline I-1 & $2,340^{\mathrm{a}}$ & $1,000^{\mathrm{a}}$ & $208{ }^{a}$ & $16,160^{\mathrm{a}}$ & $8,880^{\mathrm{a}}$ & $3,700^{\mathrm{a}}$ & $5,720^{\mathrm{a}}$ & $3,660^{\mathrm{a}}$ & $6,900^{\mathrm{a}}$ & $9,940^{\mathrm{a}}$ \\
\hline$(n=5)$ & $\pm 0,26$ & \pm 0 & $\pm 16,89$ & $\pm 0,829$ & $\pm 1,388$ & $\pm 0,282$ & $\pm 1,53$ & $\pm 0,456$ & $\pm 0,973$ & $\pm 0,973$ \\
\hline $\mathrm{I}-2$ & $10,760^{\mathrm{a}}$ & $3,429^{a}$ & $142^{\mathrm{b}}$ & $29,952^{\mathrm{b}}$ & $17,852^{\mathrm{b}}$ & 6,132 & $9,826^{\mathrm{b}}$ & $5,832^{\mathrm{a}}$ & $9,78^{\mathrm{a}}$ & $18,9^{b}$ \\
\hline$(n=5)$ & $\pm 5,485$ & $\pm 0,787$ & $\pm 14,405$ & $\pm 3,765$ & $\pm 3,144$ & $\pm 0,85$ & $\pm 1,358$ & $\pm 1,04$ & $\pm 1,751$ & $\pm 3,307$ \\
\hline I-3 & $23,800^{b}$ & $6,500^{\mathrm{a}}$ & $90,6^{\mathrm{c}}$ & $41,072^{\mathrm{C}}$ & $25,2^{c}$ & $8,193^{b}$ & $12,62^{b}$ & $7,86^{\mathrm{b}}$ & $14,046^{\mathrm{b}}$ & $23,396^{\mathrm{c}}$ \\
\hline$(n=5)$ & $\pm 1,351$ & $\pm 0,548$ & $\pm 25,774$ & $\pm 4,13$ & $\pm 4,16$ & $\pm 0,585$ & $\pm 1,31$ & $\pm 1,078$ & $\pm 2,953$ & $\pm 0,692$ \\
\hline I-4 & $27,975^{b}$ & $10,75^{a}$ & $92,667^{c}$ & $43,839^{c}$ & $28,056^{\mathrm{c}}$ & $9,344^{\mathrm{b}}$ & $13,233^{b}$ & $9,111^{\mathrm{b}}$ & $15,494^{\mathrm{b}}$ & $24,428^{\mathrm{C}}$ \\
\hline$(n=6)$ & $\pm 5,898$ & $\pm 0,5$ & $\pm 16,379$ & $\pm 2,879$ & $\pm 3,17$ & $\pm 1,199$ & $\pm 1,12$ & $\pm 1,315$ & $\pm 3,594$ & $\pm 2,241$ \\
\hline II & $28,943^{b}$ & $20,800^{\mathrm{b}}$ & $104,632^{c}$ & $43,950^{c}$ & $27,635^{\mathrm{c}}$ & $9,335^{\mathrm{b}}$ & $13,680^{\mathrm{C}}$ & $9,205^{\mathrm{b}}$ & $14,660^{\mathrm{b}}$ & $26,241^{\mathrm{C}}$ \\
\hline$(n=20)$ & $\pm 4,832$ & $\pm 8,697$ & $\pm 20,293$ & $\pm 2,458$ & $\pm 2,431$ & $\pm 1,019$ & $\pm 1,878$ & $\pm 0,873$ & $\pm 1,578$ & $\pm 2,414$ \\
\hline Grupos & $\begin{array}{l}\text { Peso } \\
(\mathrm{kg})\end{array}$ & $\begin{array}{l}\text { Idade } \\
\text { (meses) }\end{array}$ & $\mathrm{AE}$ & AE/Ao & $\mathrm{dES}$ & $\begin{array}{l}\text { SIVd/ } \\
\text { PVEd }\end{array}$ & $\begin{array}{l}\text { SIVs/ } \\
\text { PVEs }\end{array}$ & TEVE & $\mathrm{FE}$ & Vecf \\
\hline $\mathrm{I}-1$ & $2,340^{\mathrm{a}}$ & $1,000^{\mathrm{a}}$ & $11,620^{\mathrm{a}}$ & $1,166^{\mathrm{a}}$ & $1,720^{\mathrm{a}}$ & $1,019^{\mathrm{a}}$ & $0,833^{\mathrm{a}}$ & & $80,200^{\mathrm{a}}$ & \\
\hline$(n=5)$ & $\pm 0,26$ & \pm 0 & $\pm 0,3$ & $\pm 0,3$ & $\pm 0,701$ & $\pm 0,106$ & $\pm 0,232$ & $\ldots$ & $\pm 7,225$ & $\ldots$ \\
\hline $\mathrm{I}-2$ & $10,760^{\mathrm{a}}$ & $3,429^{\mathrm{a}}$ & $18,366^{\mathrm{b}}$ & $0,978^{\mathrm{a}}$ & $2,130^{\mathrm{a}}$ & $1,057^{\mathrm{a}}$ & $1,011^{\mathrm{a}}$ & $179,000^{\mathrm{a}}$ & $74,120^{\mathrm{a}}$ & $0,002^{\mathrm{a}}$ \\
\hline$(n=5)$ & $\pm 5,485$ & $\pm 0,787$ & $\pm 2,739$ & $\pm 0,09$ & $\pm 1,398$ & $\pm 0,052$ & $\pm 0,08$ & $\pm 29,808$ & $\pm 3,748$ & \pm 0 \\
\hline I-3 & $23,800^{b}$ & $6,500^{a}$ & $25,433^{\mathrm{C}}$ & $1,085^{\mathrm{a}}$ & $4,318^{\mathrm{a}}$ & $1,053^{\mathrm{a}}$ & $0,929^{a}$ & $186,750^{\mathrm{a}}$ & $66,880^{\mathrm{a}}$ & $0,002^{\mathrm{a}}$ \\
\hline$(n=5)$ & $\pm 1,351$ & $\pm 0,548$ & $\pm 3,1$ & $\pm 0,108$ & $\pm 1,696$ & $\pm 0,119$ & $\pm 0,220$ & $\pm 38,647$ & $\pm 11,734$ & \pm 0 \\
\hline $\mathrm{I}-4$ & $27,975^{b}$ & $10,75^{a}$ & $27,983^{\mathrm{C}}$ & $1,150^{\mathrm{a}}$ & $4,209^{\mathrm{a}}$ & $1,029^{\mathrm{a}}$ & $0,894^{\mathrm{a}}$ & $207,533^{\mathrm{a}}$ & $65,522^{\mathrm{a}}$ & $0,002^{\mathrm{a}}$ \\
\hline$(n=6)$ & $\pm 5,898$ & $\pm 0,5$ & $\pm 1,798$ & $\pm 0,173$ & $\pm 1,187$ & $\pm 0,079$ & $\pm 0,218$ & $\pm 8,272$ & $\pm 6,533$ & \pm 0 \\
\hline II & $28,943^{b}$ & $20,800^{b}$ & $28,416^{\mathrm{d}}$ & $1,086^{\mathrm{a}}$ & $5,289^{b}$ & $1,016^{\mathrm{a}}$ & $0,943^{\mathrm{a}}$ & $196,917^{\mathrm{a}}$ & $66,88^{\mathrm{a}}$ & $0,002^{\mathrm{a}}$ \\
\hline$(n=20)$ & $\pm 4,832$ & $\pm 8,697$ & $\pm 2,279$ & $\pm 0,077$ & $\pm 1,769$ & $\pm 0,077$ & $\pm 0,163$ & $\pm 47,293$ & $\pm 5,929$ & \pm 0 \\
\hline
\end{tabular}

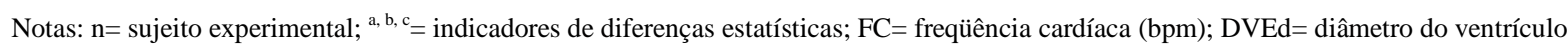
esquerdo na diástole $(\mathrm{mm})$; DVEs= diâmetro do ventrículo esquerdo na sístole (mm); SIVd= espessura do septo interventricular na diástole (mm); SIVs= espessura do septo interventricular na sístole (mm); PVEd= espessura da parede do ventrículo esquerdo na diástole (mm); PVEs= espessura da parede do ventrículo esquerdo na sístole $(\mathrm{mm}) ; \mathrm{Ao}=$ raiz da artéria aorta $(\mathrm{mm}) ; \mathrm{AE}=$ diâmetro do átrio esquerdo (mm); $\mathrm{AE} / \mathrm{Ao}$ = relação átrio E e aorta; $\mathrm{dES}$ = distância entre o ponto E da valva mitral e o SIV (mm); SIVd/PVEd= relação entre SIV e EP na diástole; SIVs/PVEs= relação entre SIV e EP na sístole; TEVE= tempo de ejeção do ventrículo esquerdo (ms); FE= fração de ejeção (\%); Vecf= velocidade de encurtamento da fibra cardíaca $(\mathrm{mm} / \mathrm{ms})$.

apresenta freqüência cardíaca de 100 (80-140) bpm; PVEd de 10 (8-12) mm; PVEs de 15 (10-19) mm; DVEd de 45(37-51) mm; DVEs de 27 (18-35) mm; FS de 39(27-55) $\%$; dES de 5 (1-10) mm; SIVd de 10 (8-13) mm; SIVs de 14 (10-17) mm; Ao de 24 (14-37) mm; eAE de 27 (16-32) mm. Portanto, os parâmetros ecocardiográficos em modo M, analisados nos animais do grupo II, apresentaram-se dentro dos limites de normalidade para a raça em questão.

Na ecocardiografia Doppler, os padrões de fluxo sangüíneo característicos são obtidos nas diferentes áreas valvares. Os picos de velocidade são geralmente maiores através da valva mitral (pico $\mathrm{E}$ geralmente é $\leq 0,9$ a $1,0 \mathrm{~m} / \mathrm{s}$; pico $\mathrm{A} \leq 0,6$ a $0,7 \mathrm{~m} \mathrm{~s}^{-1}$ ) do que na valva tricúspide. A velocidade pulmonar sistólica máxima é menor ou igual a $1,4-1,5 \mathrm{~m} \mathrm{~s}^{-1}$ na maioria dos cães sadios e a velocidade aórtica máxima é geralmente menor ou igual a 1,6-1,9m s ${ }^{-1}$. Alguns cães sadios podem apresentar velocidade aórtica máxima levemente acima de $2 \mathrm{~m} \mathrm{~s}^{-1}$, principalmente se estiverem sedados; entretanto, velocidades aórticas maiores que 2,1-2,2m s${ }^{-1}$ são sugestivas de obstrução no fluxo de saída. Raça, idade e peso corpóreo parecem ter pouca influência sobre esses parâmetros (WENDY, 2003). No presente estudo, todos os animais apresentaram-se dentro da normalidade quanto aos padrões de velocidade do fluxo sangüíneo nas diferentes áreas valvares, exceto quatro cães do grupo I e um cão do grupo II, que apresentaram pico E de velocidade em valva mitral discretamente elevado.

Uma vez que os padrões de fluxo sangüíneo e a velocidade podem ser avaliados com o Doppler, a detecção e a quantificação de insuficiência e estenose valvares, bem como de desvios cardíacos, também são possíveis. O fluxo sangüíneo e o débito cardíaco também podem ser estimados. De acordo com WENDY (2003), todos os animais enquadraram-se dentro da normalidade na ecocardiografia Doppler, exceto cinco que apresentaram, respectivamente, insuficiência aórtica discreta a moderada (um cão do subgrupo I-3); 
Tabela 2 - Média e desvio padrão de parâmetros ecocardiográficos de cães da raça Golden Retriever, clinicamente normais - São Paulo, 2005.

\begin{tabular}{|c|c|c|c|c|c|c|c|c|c|c|}
\hline Grupos & $\begin{array}{l}\text { Peso } \\
\text { (kg) }\end{array}$ & $\begin{array}{c}\text { Idade } \\
\text { (meses) }\end{array}$ & PPVE & PSIV & FS & DC & VOLs & VOLd & Vej & Mitral E \\
\hline $\mathrm{I}-1$ & $2,340^{\mathrm{a}}$ & $1,000^{\mathrm{a}}$ & $91,710^{\mathrm{a}}$ & $53,434^{\mathrm{a}}$ & $47,180^{\mathrm{a}}$ & $0,726^{\mathrm{a}}$ & $0,742^{\mathrm{a}}$ & $4,246^{\mathrm{a}}$ & $3,504^{\mathrm{a}}$ & $1,028^{\mathrm{a}}$ \\
\hline$(n=5)$ & $\pm 0,26$ & \pm 0 & $\pm 32,489$ & $\pm 36,38$ & $\pm 7,7$ & $\pm 0,088$ & $\pm 0,361$ & $\pm 0,653$ & $\pm 0,436$ & $\pm 0,179$ \\
\hline $\mathrm{I}-2$ & $10,760^{\mathrm{a}}$ & $3,429^{\mathrm{a}}$ & $67,989^{\mathrm{a}}$ & $61,037^{\mathrm{a}}$ & $41,680^{\mathrm{a}}$ & $3,529^{a}$ & $8,622^{a}$ & $33,922^{b}$ & $25,276^{\mathrm{b}}$ & $0,844^{\mathrm{a}}$ \\
\hline$(n=5)$ & $\pm 5,485$ & $\pm 0,787$ & $\pm 9,639$ & $\pm 17,956$ & $\pm 3,047$ & $\pm 0,792$ & $\pm 3,172$ & $\pm 8,410$ & $\pm 5,487$ & $\pm 0,17$ \\
\hline I-3 & $23,800^{b}$ & $6,500^{\mathrm{a}}$ & $77,295^{\mathrm{a}}$ & $54,081^{\mathrm{a}}$ & $38,507^{\mathrm{a}}$ & $4,715^{\mathrm{a}}$ & $20,820^{\mathrm{b}}$ & $75,974^{c}$ & $55,114^{c}$ & $0,771^{\mathrm{a}}$ \\
\hline$(n=5)$ & $\pm 1,351$ & $\pm 0,548$ & $\pm 14,3$ & $\pm 13,025$ & $\pm 7,375$ & $\pm 0,836$ & $\pm 8,232$ & $\pm 18,160$ & $\pm 16,644$ & $\pm 0,15$ \\
\hline $\mathrm{I}-4$ & $27,975^{\mathrm{b}}$ & $10,75^{\mathrm{a}}$ & $68,710^{\mathrm{a}}$ & $43,287^{\mathrm{a}}$ & $35,994^{a}$ & $5,584^{\mathrm{a}}$ & $28,981^{b}$ & $87,381^{c}$ & $58,560^{c}$ & $0,771^{\mathrm{a}}$ \\
\hline$(n=6)$ & $\pm 5,898$ & $\pm 0,5$ & $\pm 22,841$ & $\pm 18,78$ & $\pm 5,029$ & $\pm 1,479$ & $\pm 5,928$ & $\pm 14,633$ & $\pm 11,038$ & $\pm 0,152$ \\
\hline II & $28,943^{b}$ & $20,800^{\mathrm{b}}$ & $59,678^{a}$ & $46,904^{a}$ & $37,075^{a}$ & $6,059^{\mathrm{a}}$ & $28,870^{b}$ & $87,565^{c}$ & $58,835^{c}$ & $0,724^{\mathrm{a}}$ \\
\hline$(n=20)$ & $\pm 4,832$ & $\pm 8,697$ & $\pm 14,748$ & $\pm 18,218$ & $\pm 4,745$ & $\pm 1,524$ & $\pm 6,485$ & $\pm 11,213$ & $\pm 9,587$ & $\pm 0,176$ \\
\hline Grupos & $\begin{array}{l}\text { Peso } \\
(\mathrm{kg})\end{array}$ & $\begin{array}{c}\text { Idade } \\
\text { (meses) }\end{array}$ & Mitral A & Acel. & Ao Vmáx & Aо TA & $\begin{array}{c}\text { Ao } \\
\text { grad. }\end{array}$ & $\begin{array}{c}\text { Ap } \\
\text { Vmáx }\end{array}$ & $\begin{array}{c}\text { Ap } \\
\text { grad. }\end{array}$ & TRIV \\
\hline $\mathrm{I}-1$ & $2,340^{\mathrm{a}}$ & $1,000^{\mathrm{a}}$ & $0,539^{\mathrm{a}}$ & & $1,477^{\mathrm{a}}$ & $26,733^{\mathrm{a}}$ & & & & $0,041^{\mathrm{a}}$ \\
\hline$(n=5)$ & $\pm 0,26$ & \pm 0 & \pm 0 & $\ldots$ & $\pm 0,210$ & $\pm 8,064$ & $\ldots$ & $\ldots$ & $\ldots$ & $\pm 0,005$ \\
\hline $\mathrm{I}-2$ & $10,760^{\mathrm{a}}$ & $3,429^{a}$ & $0,556^{\mathrm{a}}$ & $50,000^{\mathrm{a}}$ & $1,244^{\mathrm{a}}$ & $48,133^{\mathrm{a}}$ & $6,023^{\mathrm{a}}$ & $0,930^{\mathrm{a}}$ & $3,944^{\mathrm{a}}$ & $0,073^{\mathrm{a}}$ \\
\hline$(n=5)$ & $\pm 5,485$ & $\pm 0,787$ & $\pm 0,187$ & \pm 10 & $\pm 0,090$ & $\pm 23,211$ & $\pm 0,754$ & $\pm 0,067$ & $\pm 0,672$ & $\pm 0,008$ \\
\hline I-3 & $23,800^{\mathrm{b}}$ & $6,500^{\mathrm{a}}$ & $0,569^{\mathrm{a}}$ & $72,667^{\mathrm{a}}$ & $1,321^{\mathrm{a}}$ & $40,266^{\mathrm{a}}$ & $6,200^{\mathrm{a}}$ & $1,032^{\mathrm{a}}$ & $4,337^{\mathrm{a}}$ & $0,067^{\mathrm{a}}$ \\
\hline$(n=5)$ & $\pm 1,351$ & $\pm 0,548$ & $\pm 0,06$ & $\pm 6,429$ & $\pm 0,201$ & $\pm 16,54$ & $\pm 0,967$ & $\pm 0,172$ & $\pm 1,45$ & $\pm 0,029$ \\
\hline $\mathrm{I}-4$ & $27,975^{b}$ & $10,75^{\mathrm{a}}$ & $0,441^{\mathrm{a}}$ & $61,748^{\mathrm{a}}$ & $1,299^{\mathrm{a}}$ & $49,266^{\mathrm{b}}$ & $6,963^{\mathrm{a}}$ & $1,037^{\mathrm{a}}$ & $4,546^{\mathrm{a}}$ & $0,065^{\mathrm{a}}$ \\
\hline$(n=6)$ & $\pm 5,898$ & $\pm 0,5$ & $\pm 0,062$ & $\pm 14,672$ & $\pm 0,243$ & $\pm 12,053$ & $\pm 2,823$ & $\pm 0,183$ & $\pm 1,832$ & $\pm 0,023$ \\
\hline II & $28,943^{b}$ & $20,800^{b}$ & $0,514^{\mathrm{a}}$ & $61,499^{\mathrm{a}}$ & $1,200^{\mathrm{a}}$ & $43,783^{b}$ & $6,418^{a}$ & $1,067^{\mathrm{a}}$ & $4,729^{a}$ & $0,065^{\mathrm{a}}$ \\
\hline$(n=20)$ & $\pm 4,832$ & $\pm 8,697$ & $\pm 0,133$ & $\pm 12,449$ & $\pm 0,269$ & $\pm 14,431$ & $\pm 2,775$ & $\pm 0,15$ & $\pm 1,241$ & $\pm 0,017$ \\
\hline
\end{tabular}

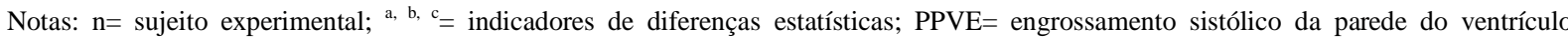
esquerdo (\%); PSIV= engrossamento sistólico do septo interventricular (\%); FS= fração de encurtamento (\%); DC= débito cardíaco (L/min); VOLs= volume na sístole $(\mathrm{mL}) ;$ VOLd= volume na diástole $(\mathrm{mL}) ; \mathrm{Vej}=$ volume de ejeção $(\mathrm{mL}) ; \mathrm{Mitral} \mathrm{E}=$ fluxo mitral onda E (m/s); Mitral A = fluxo mitral onda A (m/s); Acel.= aceleração- fluxo mitral (ms); Ao Vmáx= velocidade máxima do fluxo aórtico (m/s); Ao TA= aceleração- fluxo aórtico (ms); Ao grad. = gradiente de pressão- fluxo aórtico (mm de Hg); Ap Vmáx= velocidade máxima do fluxo pulmonar (m/s); Ap grad.= gradiente de pressão- fluxo pulmonar (mm de Hg); TRIV= tempo de relaxamento isovolumétrico (s).

escape discreto em mitral (um cão do subgrupo I-4, e dois do grupo II); e insuficiência pulmonar discreta e espessamento de valva mitral (um cão do grupo II).

De acordo com os resultados obtidos no presente estudo, os valores ecocardiográficos de referência para cães da raça Golden Retriever de até 12 meses de idade e peso corpóreo médio de 15,6 \pm 10,998kg são: $F C=131,3 \pm 51,5 b p m ; D V E d=33,2 \pm$ 11,5mm; DVEs= 20,3 \pm 8,153mm; $\mathrm{SIVd}=6,9 \pm 2,3 \mathrm{~mm}$; SIVs $=10,4 \pm 3,2 \mathrm{~mm} ; \mathrm{PVEd}=6,7 \pm 2,3 \mathrm{~mm} ; \mathrm{PVEs}=11,7 \pm$ 4,2mm; $\mathrm{Ao}=19,4 \pm 6,1 \mathrm{~mm} ; \mathrm{AE}=21,1 \pm 7,0 \mathrm{~mm} ; \mathrm{AE} / \mathrm{Ao}=$ $1,0 \pm 0,1 ; \mathrm{dES}=3,1 \pm 1,6 \mathrm{~mm} ; \mathrm{SIVd} / \mathrm{PVEd}=1,0 ; \mathrm{SIVs} /$ PVEs $=0,9 \pm 0,1 ;$ TEVE $=191,4 \pm 28,3 \mathrm{~ms} ; \mathrm{FE}=71,3 \pm$ 9,3\%; Vecf $=0,002 \mathrm{~mm} \mathrm{~ms}^{-1}$; PPVE $=76 \pm 22,2 \%$; PSIV= $52,4 \pm 22,2 \%$; FS = 40,6 $\pm 7 \%$; DC= 3,6 $\pm 2 \mathrm{~L} \mathrm{~min}^{-1}$; VOLs= $14,7 \pm 12,1 \mathrm{~mL} ; \mathrm{VOLd}=50,3 \pm 35,9 \mathrm{~mL} ; \mathrm{Vej}=35,6 \pm 25 \mathrm{~mL}$; Mitral $\mathrm{E}=0,85 \pm 0,18 \mathrm{~m} \mathrm{~s}^{-1}$; Mitral A=0,51 $\pm 0,12 \mathrm{~m} \mathrm{~s}^{-1}$; Aceleração= 61,4 $\pm 13,7 \mathrm{~ms}$; Ao Vmáx $=1,33 \pm 0,2 \mathrm{~m} \mathrm{~s}^{-1}$; Ao TA=41,4 \pm 17,1ms; Ao grad.= 6,44 $\pm 1,84 \mathrm{~mm}$ de Hg; Ap Vmáx $=1 \pm 0,14 \mathrm{~m} / \mathrm{s}$; Ap grad= 4,28 $\pm 1,36 \mathrm{~mm}$ de Hg;
TRIV $=0,06 \pm 0,02 s$. E os valores ecocardiográficos de referência para cães da raça Golden Retriever de 12 a 36 meses de idade e peso corpóreo médio de 28,943 \pm 4,832 kg são: $\mathrm{FC}=104,6 \pm 20,2 \mathrm{bpm}$; $\mathrm{DVEd}=43,9 \pm 2,4 \mathrm{~mm}$; DVEs $=27,6 \pm 2,4 \mathrm{~mm}$; SIVd= 9,3 $\pm 1 \mathrm{~mm}$; SIVs= 13,6 \pm 1,8mm; PVEd= 9,2 $\pm 0,8 \mathrm{~mm}$; PVEs $=14,6 \pm 1,5 \mathrm{~mm} ; \mathrm{Ao}=$ $26,2 \pm 2,4 \mathrm{~mm} ; \mathrm{AE}=28,4 \pm 2,2 \mathrm{~mm} ; \mathrm{AE} / \mathrm{Ao}=1 ; \mathrm{dES}=5,2 \pm$ 1,7mm; SIVd/PVEd= 1; SIVs/PVEs= 0,9 $\pm 0,1$; TEVE= $196,9 \pm 47,2 \mathrm{~ms} ; \mathrm{FE}=66,8 \pm 5,9 \%$; Vecf $=0,002 \mathrm{~mm} \mathrm{~ms}^{-1}$; $\mathrm{PPVE}=59,6 \pm 14,7 \%$; PSIV $=46,9 \pm 18,2 \% ; \mathrm{FS}=37 \pm$ 4,7\%; DC=6 $\pm 1,5 \mathrm{~L} \mathrm{~min}^{-1}$; VOLs $=28,8 \pm 6,4 \mathrm{~mL}$; VOLd= $87,5 \pm 11,2 \mathrm{~mL} ; \mathrm{Vej}=58,8 \pm 9,5 \mathrm{~mL} ;$ Mitral $\mathrm{E}=0,75 \pm 0,17 \mathrm{~m}$ $\mathrm{s}^{-1}$; Mitral A = 0,51 $\pm 0,13 \mathrm{~m} \mathrm{~s}^{-1}$; Aceleração $=61,4 \pm$ 12,4ms; Ao Vmáx $=1,2 \pm 0,2 \mathrm{~m} \mathrm{~s}^{-1}$; Ao TA= 43,7 \pm 14,4ms; Ao grad.=6,41 $\pm 2,75 \mathrm{~mm}$ de Hg; Ap Vmáx $=1 \pm 0,15 \mathrm{~m} \mathrm{~s}^{-1}$; Ap grad= 4,72 $\pm 1,24 \mathrm{~mm}$ de Hg; TRIV = 0,06 $\pm 0,01 \mathrm{~s}$.

\section{CONCLUSÕES}

Na análise dos resultados obtidos, por meio da metodologia utilizada no presente estudo, 
fundamenta-se que cães da raça Golden Retriever apresentam alteração significativa no peso corpóreo a partir de três a seis meses de idade, influenciando alguns parâmetros ecocardiográficos. O peso corpóreo tem influência positiva sobre a maioria dos parâmetros ecocardiográficos em modo $\mathrm{M}$, mas não influencia a maior parte dos parâmetros da ecocardiografia Doppler. Além disso, os parâmetros ecocardiográficos são passíveis de variação dentro de uma mesma raça canina.

\section{AGRADECIMENTOS}

À FAPESP, pela concessão da bolsa de Iniciação Científica (processo $n^{\circ}$ : 04/08173-2); à Prof ${ }^{a} \operatorname{Dr}^{\mathrm{a}}$ Maria Angélica Miglino, Professora Titular do Departamento de Cirurgia da Faculdade de Medicina Veterinária e Zootecnia da Universidade de São Paulo (FMVZ-USP), pela cessão dos cães do canil GRMDBrasil; à Prof ${ }^{\mathrm{a}} \mathrm{Dr}^{\mathrm{a}}$ Mayana Zatz, Professora Titular do Centro de Estudos do Genoma Humano do Instituto de Biociências da Universidade de São Paulo, pela realização da genotipagem dos animais; aos médicos veterinários e pós-graduandos que colaboraram com o projeto, Fernanda Lie Yamaki, Roberto Carvalho e Pereira, André Luís Fernandes dos Santos e Valéria Costa Marinho de Oliveira; aos proprietários particulares; ao Canil Fortaleza da Aldeia (Carapicuíba-SP); ao Canil GRMDBrasil (FMVZ-USP) e seus proprietários cadastrados; ao Canil Golden Trip (Granja Viana-SP); ao Canil de Golden Retriever (São Roque-SP); e ao Canil de Golden Retriever (Itu-SP), pela participação no presente projeto.

\section{REFERÊNCIAS}

ANDERSON, J.L. et al. Brain function in Duchenne muscular dystrophy. Brain, v.125, p.4-13, 2002.

BOGDANOVICH, S. et al. Therapeutics for Duchenne muscular dystrophy: current approaches and futures directions. Journal of Molecular Medicine, v.82, p.102-115, 2003.

BOON, J.A. Manual of veterinary echocardiography. Baltimore: Williams \& Wilkins, 1998. 478p.

CHETBOUL, V. et al. Tissue doppler imaging detects early asymptomatic myocardial abnormalities in a dog model of Duchenne's cardiomyopathy. European Heart Journal, v.25, p.1934-1939, 2004.

CHILDERS, M.K. et al. Eccentric contraction injury in dystrophic canine muscle. Archives of Physical Medicine and Rehabilitation, v.83, p.1572-1578, 2002.

COLLINS, C.A.; MORGAN, J.E. Duchenne's muscular dystrophy: animal models used to investigate pathogenesis and develop therapeutic strategies. International Journal of Experimental Pathology, v.84, p.165-172, 2003.

COZZI, F. et al. Development of muscle pathology in canine X-linked muscular dystrophy. Quantitative characterization of histopathological progression during postnatal skeletal muscle development. Acta Neuropathology, v.101, p.469478, 2001.

CZINER, D.G.; LEVIN, R.I. The cardiomyopathy of Duchenne's muscular dystrophy and the function of dystrophin. Medical Hypotheses, v.40, p.169-173, 1993.
DANI, S.U. et al. Establishment of a muscular dystrophy dog colony in Brazil. Genetics and Molecular Biology, v.23, n.3, supplement, p.75, 2000.

FOX, P.R. et al. Textbook of canine and feline cardiology: principles and clinical practice. 2.ed. Philadelphia: Saunders, 1999. 955p.

HAINSEY, T.A. et al. Cardiomyopathic features associated with muscular dystrophy are independent of dystrophin absence in cardiovasculature. Neuromuscular Disorders, v.13, p.294302, 2003.

HOOGERWAARD, E.M. et al. Cardiac involvement in carriers of Duchenne and Becker muscular dystrophy. Neuromuscular Disorders, v.9, p.347-351, 1999.

HOWELL, J.M. et al. Use of the dog model for Duchenne muscular dystrophy in gene therapy trials. Neuromuscular Disorders, v.7, p.325-328, 1997.

HUNSAKER, R.H. et al. Cardiac function in Duchennes's muscular dystrophy. American Journal of Medicine, v.73, p.235-238, 1982.

KAMOGAWA, Y. et al. Dystrophyn-deficient myocardium is vulnerable to pressure overload in vivo. Cardiovascular Research, v.50, p.509-515, 2001.

MOISE, N.S. et al. Duchenne's cardiomyopathy in a canine model: electrocardiographic and echocardiographic studies. Journal of the American College of Cardiology, v.17, p.812-820, 1991.

NGUYEN, F. et al. Muscle lesions associated with dystrophin deficiency in neonatal Golden Retriever puppies. Journal of Comparative Pathology, v.126, p.100-108, 2002.

NOLAN, M.A. et al. Cardiac assessment in childhood carriers of Duchenne and Becker muscular dystrophies. Neuromuscular Disorders, v.13, p.129-132, 2003.

PACIORETTY, L.M. et al. Cellular and molecular biology: reduction of the transient outward potassium current in canine X-linked muscular dystrophy. Circulation, v.90, n.3, p.13501356, 1994.

SHIMATSO, Y. et al. Canine X-linked muscular dystrophy in Japan (CXMDj). Experimental Animal, v.52, n.2, p.93-97, 2003.

THOMAS, W.P. et al. Recommendations for standards in transthoracic two-dimensional echocardiography in the dog and cat. Journal of Veterinary Internal Medicine, v.7, n.4, p.247-252, 1993.

VALENTINE, B.A. et al. Canine X-linked muscular dystrophy as an animal model of Duchenne muscular dystrophy: a review. American Journal of Medical Genetics, v.42, n.3, p.352356, 1992.

WENDY, A.W. Diagnostics tests for the cardiovascular system. In: NELSON, R.W.; COUTO, C.G. Small animal internal medicine. 3.ed. Sant Louis Missouri: Mosby, 2003. Cap.2, p.12-50.

YAMATO, R.J. Estudo dos parâmetros ecocardiográficos em modo $\mathrm{M}$ de cães da raça Poodle miniatura clinicamente sadios. Ciência Rural, v.36, n.1, p.142-148, 2006. 